\title{
Connections between whistlers and pulsation activity
}

\author{
J. Verö ${ }^{1}$, B. Zieger ${ }^{1}$, J. Szendröi ${ }^{1}$, M. Vellante ${ }^{2}$, J. Střesğtik ${ }^{3}$, H. Lühr ${ }^{4}$, A. Best ${ }^{5}$, A. Körmendi ${ }^{6}$ \\ J. Lichtenberger ${ }^{7}$, T. Ménesi ${ }^{1}$, P. Bencze ${ }^{1}$, F. Märcz ${ }^{1}$, V. Wesztergom ${ }^{1}$ \\ ${ }_{1}^{1}$ Geodetic and Geophysical Research Institute of the Hungarian Academy of Sciences, H-9401 Sopron, Pf. 5, Hungary \\ ${ }^{2}$ Dipartimento di Fisica, Universita' dell'Aquila, Via Vetoio, I-67010 Coppito-L'Aquila, Italy \\ ${ }^{3}$ Geofysikalni Ústav, AV ČR, Praha 4, Spořilov. Bočni II 1401, CZ-14131, Czech Republic \\ ${ }_{5}^{4}$ GeoForschungsZentrum Potsdam, Telegrafenberg, D-14473 Potsdam, Germany \\ ${ }^{5}$ GeoForschungsZentrum Potsdam, Adolf Schmidt Geomagnetisches Observatorium D-14823 Niemegk, Germany \\ ${ }^{6}$ Loránd Eötvös Geophysical Institute, H-1440 Budapest, Pf. 35, Hungary \\ ${ }^{7}$ Loránd Eötvös University, Institute of Geophysics, H-1083 Budapest, Ludovika tér 2, Hungary
}

Received: 11 October 1999 / Revised: 15 March 2000 / Accepted: 4 April 2000

\begin{abstract}
Simultaneous whistler records of one station and geomagnetic pulsation $(\mathrm{Pc} 3)$ records at three stations were compared. In a previous study correlation was found between occurrence and $L$ value of propagation/excitation for the two phenomena. The recently investigated simultaneous records have shown that the correlation is better on longer time scales (days) than on shorter ones (minutes), but the $L$ values of the propagation of whistlers/excitation of pulsations are correlated, i.e. if whistlers propagate in higher latitude ducts, pulsations have periods longer than in the case when whistlers propagate in lower latitude ducts.
\end{abstract}

Key words: Electromagnetics (wave propagation) Magnetospheric physics (magnetospheric configuration and dynamics; MHD waves and unstabilities)

\section{Introduction}

Whistlers and geomagnetic pulsations from field line resonances are two phenomena which need a certain magnetospheric structure for excitation. Whistlers propagate from one hemisphere to the other in ducts, i.e. in tubes which are elongated approximately along geomagnetic field lines and are bordered by some inhomogeneity in the particle density. The main factors governing whistler activity are propagation conditions from the source (lightning) to the magnetospheric channel and then the existence of appropriate channels in the magnetosphere which lead them to the opposite hemisphere (Maeda and Kimura, 1956; Smith, 1960; Smith et al., 1960; Smith and Angerami, 1968; Helliwell, 1965; Walker, 1976, 1978; Strangeways, 1976, 1982).

Correspondence to: J. Verö

e-mail: vero@ggki.hu
Field line resonances are excited by waves/inhomogeneities arriving into the magnetosphere from the interplanetary space. Due to these upstream waves, shells (whether they are of finite or infinitesimal thickness) are excited with a frequency characteristic for the given shell (Orr, 1976; Yumoto, 1986; Cz Miletits et al., 1990). These waves are observed at mid-latitudes at the surface as Pc3 pulsations. Again, the possibility of the excitation of field line resonances depends on several factors which are partly unknown at present. One of these factors is surely a not too high particle density in the magnetosphere, as during solar maximum conditions when particle density exceeds a certain level field line resonances do not occur, or are at least very seldom excited (Verö and Menk, 1986; Verö, 1996).

Even if the two magnetospheric structures are different, both need some kind of inhomogeneity along geomagnetic field lines, in the case of whistlers, linear structures along the field lines, in the case of field line resonances, shell-like structures on surfaces of equal $L$-values. Such shell-like structures if not infinitely thin may correspond to a series of ducts along this surface (Takahashi et al., 1993). This is the background for a possible correlation of whistler and pulsation activities.

There are several experimental facts which suggest a connection between whistler and pulsation activities. At first, a direct (and for us the only known mid-latitude) study of the whistler and pulsation activities (Verö et al., 1997) has shown two kinds of connections. The spectra of (Pc3-4) geomagnetic pulsations are different in times when whistlers propagate at low and at high $L$-values. In the latter case periods are longer, corresponding to the resonant period of the field line along which whistlers propagated, in the former case they are shorter. Additionally, a statistical analysis of whistler and pulsation activities has shown that pulsation activity is higher on days when the whistler activity is high and vice versa. Daytime pulsation activity is correlated both with daytime and night-time whistler activities.

This preliminary study was a statistical one in both respects. In the case of the connection between $L$-value 
of the whistler ducts (recorded at the Tihany, THY observatory) and pulsation periods (recorded at Nagycenk, NCK, about $120 \mathrm{~km}$ away), parameters deduced from whistlers were compared to the pulsation activity of simultaneous $30 \mathrm{~min}$ long intervals as found in the NCK pulsation catalogue. Data of about 1700 whistlers were used in this comparison. A strict simultaneity could not be ensured, only the $30 \mathrm{~min}$ long intervals of the whistler appearance were the same for the pulsation activity.

In the comparison of whistler occurrence frequency and pulsation activity, the observed number of whistlers (Panska Ves, PAV observatory in the Czech Republic, about $200 \mathrm{~km}$ away) in a two-hour long daily and a onehour long night interval was compared to the NCK daily index of pulsation activity. In this case, the strict simultaneity of data was even less certain, only longer time averages (one day) were used, thus features (whistler ducts and field line shells) persisting for at least one day could be compared.

Recently Smith et al. (1998) found correlation between Antarctic (geomagnetic and VLF/ELF) Pc3 pulsations and whistlers. According to their results, a part of the QP (quasi-periodic) emissions with periods of 10-60 s, known as type I QPs are linked with geomagnetic pulsations and correspond to upstream waves. Type II QPs are uncorrelated with geomagnetic pulsations, and are always accompanied by shorter period (4-6 s) VLF/ELF PEs (periodic emissions).

Another line of evidence suggesting extraterrestrial influences in whistler activity is presented in Fig. 1. It shows the dependence of the observed yearly average number of whistlers per minute (PAV) on the (yearly average intensity of the) interplanetary magnetic field (IMF) based on 11 years of data. In spite of a significant scatter, an influence of IMF is evidently present in whistler activity, perhaps due to an influence on the source mechanism, or due to an effect on propagation (the correlation coefficient is -0.49 between the two sets, the number of whistlers decreases by 0.4 per minute for an increase of $1 \mathrm{nT}$ in IMF scalar magnitude; the

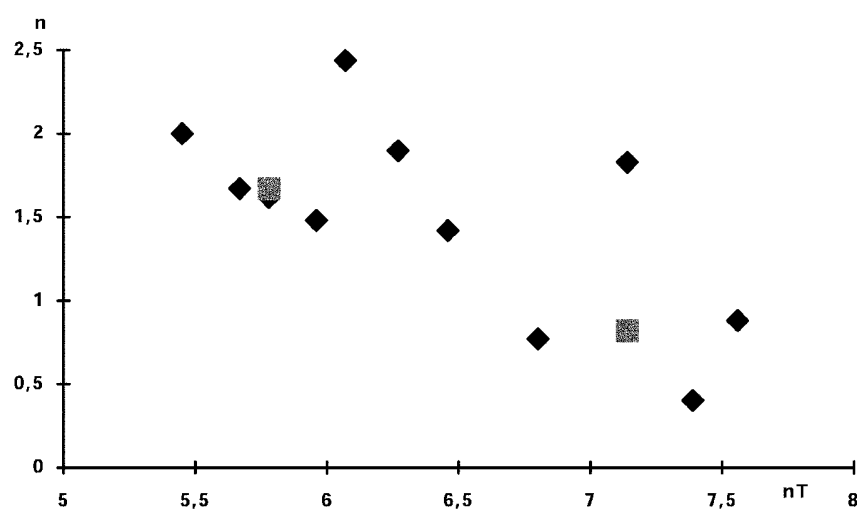

Fig. 1. Average number of whistlers per minute (n, diamonds) in the years 1971-1979, 1987 and 1990 versus the (yearly average) intensity of the interplanetary magnetic field (B). Medians of the whistler numbers for high and low B values (limit $6.5 \mathrm{nT}$ ) are indicated by squares averages of the two groups differ according to the Student-test on a level of significance of 0.99).

The aim of the present study of pulsations at three (L'Aquila, LAQ, $L \sim 1.59$, Nagycenk, NCK, $L \sim 1.91$, Niemegk, NGK, $L \sim 2.29$ at a height of $200 \mathrm{~km}, 1.53$, $1.85,2.22$ at the surface) observatories and of whistlers at one station (Tihany, THY, $L \sim 1.86$ and 1.80 ) was to study the connection in detail, i.e. in shorter time intervals. We also had pulsation records from Budkov, but due to malfunction of the recorder, we did not use them.

\section{Planning the simultaneous measurements}

The task of the selection of the daily time interval for the simultaneous recording of geomagnetic pulsations and whistlers is a difficult one. As the digital whistler recording system in Tihany did not allow us to record in intervals longer than one hour daily, and even this was at the limit of its capabilities, we had to select the most advantageous one hour section of the day. Figures 2 and 3 represent the daily distribution of whistlers and pulsations, in different seasons. On the basis of these distributions, we selected the interval 0300 to 0400 UT, i.e. $4 \mathrm{~h}$ to $5 \mathrm{~h}$ LT for the recording. During this interval, the number of whistlers is about one third of the

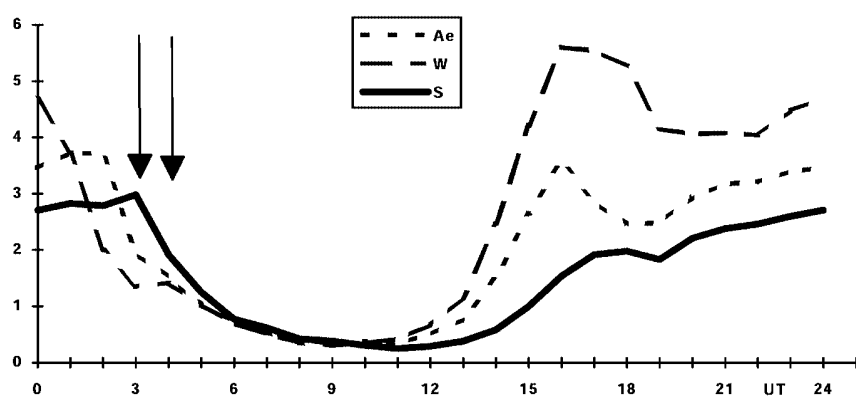

Fig. 2. Daily variation of the number of whistlers per minute in different seasons ( $S$ summer, May to August, $W$ winter, November to February, Ae equinox, March, April, September, October), at the Observatory Panska Ves, 1971-79, 1987 and 1990. The selected interval for the present study is indicated by arrows. LT is UT $+1 \mathrm{~h}$ approximately

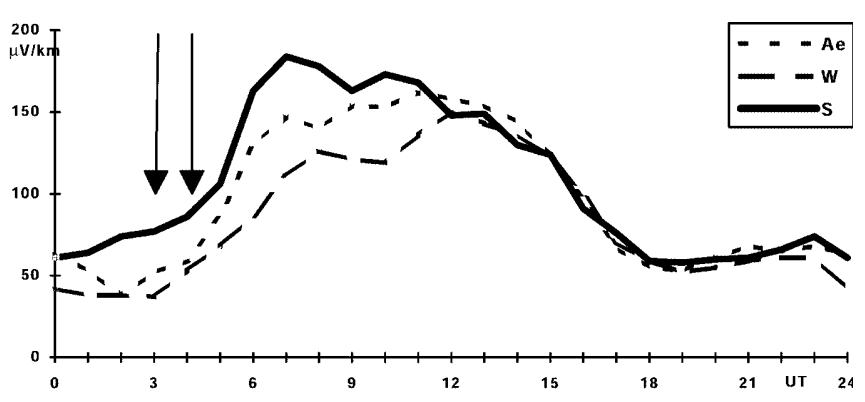

Fig. 3. Daily variation of the average pulsation amplitudes $(\mu \mathrm{V} / \mathrm{km})$ in different seasons (for periods shorter than $2 \mathrm{~min}$ ), in long-time average from the Nagycenk Observatory (1957-1991). The selected interval for the present study is indicated by arrows 


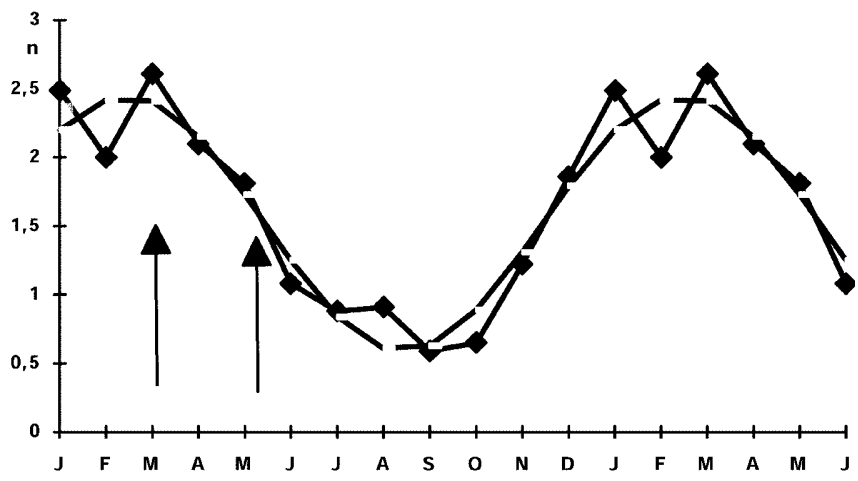

Fig. 4. Yearly variation of the number of whistlers per minute (Panska Ves) on average for the 11-year cycle and a sine-wave with a period of one year fitted to it with maxima on March 1 and a minimum on September 1. The interval selected for the present study is indicated by arrows

maximum occurrence frequency, and the amplitude of (Pc3) pulsations is about half of the daily maximum. The "product" of the two, however, is maximum during this interval. Afternoon intervals were excluded from the selection, as pulsations during the afternoon hours have slightly different characteristics according to previous experience in the sense that field line resonant (i.e. regularly sinusoidal) events occurred less frequently and therefore selected intervals from afternoon hours in earlier arrays were correspondingly much less numerous than morning ones in spite of equal probabilities of the two groups (Verö et al., 1998).

The second question concerned the selection of the yearly location of the measurements. The number of whistlers has (Fig. 4) a yearly wave with maximum around March 1 and minimum around September 1 . Pulsations have, at least during minimum, solar activity, only a small maxima at equinoxes. Thus the most advantageous interval was (considering weather conditions also) from mid-March to mid-April and this interval was prolonged in both directions by a few weeks. At some stations, including THY whistlers, the interval of recording was even prolonged till mid-June, but the results from this prolongation were very poor, since simultaneous whistlers and pulsations were recorded only in a few cases in early June.

Due to service difficulties, records were only made on working days (Monday to Friday): records were saved if an audio checking of the digital whistler records detected whistlers, this condition led to a loss of about $80 \%$ of the recorded 60 intervals. Thus we obtained a sample consisting of 12 one hour long simultaneous records of both phenomena, whistlers at THY, pulsation at LAQ, NCK and NGK. The total number of whistlers during these days was 200 , corresponding to roughly 0.3 whistlers/min, being less than expected (about 1 whistler/min) in spite of the fact that only records with identifiable whistler activity were kept.

This somewhat lengthy description of the experimental conditions was necessary in order to explain the amount and quality of data. We also tried to get whistler records from other stations, but failed. Apparently, no group is able to record whistlers digitally with similar parameters to those in Tihany. Whistler recording has been suspended in Panska Ves, too, from where we obtained valuable data previously.

\section{Amount and quality of data used in the investigation}

As mentioned previously, we had simultaneous onehour long records for 12 days, but in the following, only six of them will be used due to different reasons (too few whistlers, too low pulsation activity, difficulties with the identification of whistlers due to higher noise level etc.).

The whistlers identified in the records were then analysed at the Geophysical Department of the Eötvös Loránd University, Budapest, using Tarcsai's (1975) programme. The lowest limit of the applicability of this programme is $L \approx 1.5$, the results are $L$-value of the propagation, electron density in the equatorial plane and tube content (of electrons). In the following, mainly $L$-values will be used.

The observed number of whistlers along different $L$-values, the geomagnetic activity and the magnitude of the interplanetary magnetic field on these days is given in Table 1.

Table 1 also includes the two days on which most, i.e. 3 and 1 whistlers were observed but were not included in this study. About half of all whistlers occurred on a single day (April 4). The average $K p$ index for whistlers propagating at different $L$-values does not differ very significantly, since all $K p$-averages are within the interval 3 to 3.8 , nevertheless, there is a slight tendency for higher $K p$ values if whistlers propagated at lower $L$-values.
Table 1. Data on the recorded whistlers

\begin{tabular}{lcccccccc}
\hline Day & Whistlers & $L 2.5$ & $L 2.5-2.75$ & $L 2.75-3.0$ & $L 3.0-$ & $K p$ & Average $L$ & IMF, nT \\
\hline March 17 & $\mathbf{6}$ & $\mathbf{0}$ & $\mathbf{0}$ & $\mathbf{2}$ & $\mathbf{4}$ & $\mathbf{1}+$ & $\mathbf{3 . 1}$ & $\mathbf{4}-\mathbf{5}$ \\
March 18 & 3 & 0 & 2 & 0 & 1 & $3-$ & 2.9 & $5-6$ \\
March 30 & 1 & 0 & 0 & 0 & 1 & $4-$ & 3.2 & $4-6$ \\
April 04 & $\mathbf{9 2}$ & $\mathbf{3 7}$ & $\mathbf{3 2}$ & $\mathbf{1 8}$ & $\mathbf{5}$ & $\mathbf{3}+$ & $\mathbf{2 . 6}$ & $\mathbf{5}-\mathbf{1 0}$ \\
April 05 & $\mathbf{4 4}$ & $\mathbf{1}$ & $\mathbf{1 5}$ & $\mathbf{1 9}$ & $\mathbf{9}$ & $\mathbf{3}+$ & $\mathbf{2 . 8}$ & $\mathbf{3}-\mathbf{5}$ \\
April 17 & $\mathbf{3 0}$ & $\mathbf{4}$ & $\mathbf{2 0}$ & $\mathbf{4}$ & $\mathbf{2}$ & $\mathbf{5}+$ & $\mathbf{2 . 6}$ & $\mathbf{1 0}-\mathbf{1 2}$ \\
April 19 & $\mathbf{8}$ & $\mathbf{2}$ & $\mathbf{5}$ & $\mathbf{1}$ & $\mathbf{0}$ & $\mathbf{2}+$ & $\mathbf{2 . 6}$ & $\mathbf{5}-\mathbf{7}$ \\
June 07 & $\mathbf{4}$ & $\mathbf{0}$ & $\mathbf{1}$ & $\mathbf{3}$ & $\mathbf{0}$ & $\mathbf{3}+$ & $\mathbf{2 . 8}$ & $\mathbf{6 - 8}$ \\
Cases & 188 & 44 & 75 & 47 & 22 & & & \\
Average $K p$ & 3.5 & $3.5(3+)$ & $3.8(4-)$ & $3.4(3+)$ & $3.0(3)$ & & $L \approx 2.7$ & \\
\hline
\end{tabular}

Bold numbers indicate days used in the present study, thin ones the next two days with most whistlers 


\section{Connection between the occurrence of whistlers and pulsation activity}

The previous study cited in the Introduction (Verö et al., 1997) concluded that there is a connection between the number of whistlers and the simultaneous activity of Pc3 type geomagnetic pulsations. This connection was found valid for the number of both night-time and day-time whistlers, with the restriction that whistler numbers were taken from five minute records at the beginning of two and three night- and day-time hours, respectively, and pulsations amplitudes were activity indices for whole days, based on daily average amplitudes of all pulsations having periods shorter than $2 \mathrm{~min}$. Considering all the restrictions from these data selections, the connection was confirmed between long-time (daily) pulsation activity and whistler occurrence. The dominant role in the pulsation activity as defined previously is from Pc3 pulsations, and here the more important influence is from the actual possibility for the excitation of field line resonances, as interplanetary sources barely influence directly a magnetospheric process such as whistler propagation. Thus, the connection observed previously exists between the possibility of exciting field line resonances and the occurrence of whistlers, with time scales of one hour or more, comparable to the lifetime of both field line resonant structures and whistler ducts. Nothing is known, however, about the short-term connection between the two phenomena. Therefore we tried to investigate short-time indices of whistler activity. In the present case this was the number of whistlers during each 1 min section in the recorded time interval of $1 \mathrm{~h}$, with the pulsation activity due to field line resonance in the three observatories during the same $1 \mathrm{~min}$ long interval. The field line resonant periods have been selected according to the following terms.

On average, the field line resonance has an average period of $15 \mathrm{~s}$ at LAQ, $22 \mathrm{~s}$ at NCK and $35 \mathrm{~s}$ at NGK. During solar minimum conditions we found previously shorter-than-average field line resonant periods (Verö, 1996). That means that periods could be shorter by about $10 \%$ than the average in 1997 . However, there is also a daily change of the field line resonant period (Lee, 1982; Poulter et al., 1984) which fact was also confirmed by experimental data of NCK. This would mean periods shorter by $20 \%$ than the average in the interval $4-5 \mathrm{~h}$ LT. Thus finally, the field line resonant period was presumed to be 10 to $12 \mathrm{~s}$ at LAQ, 18 to $20 \mathrm{~s}$ at NCK and 27 to $29 \mathrm{~s}$ at NGK.

The data for the comparison were obtained by the following method: in each minute an amplitude value was selected for the FLR type pulsations from dynamic spectra. If there was an amplitude maximum in the actual minute in the mentioned period range, then this maximum was accepted as amplitude. If no maximum was found in the actual minute in the FLR range, then the average amplitude in the given minute and period range was used. As the purpose of the present study was not to compare activities at different observatories, amplitudes were used as relative values and in the final comparison, the level of reference was the most frequently occurring amplitude bin (bins were fixed at steps of 1 in binary logarithms, i.e. the ratio of the limits is 2).

In Fig. 5, results for two intervals are represented from the six chosen. The first, April 4, 1997 was the day when most whistlers were recorded, while on March 17, 1997, the connection between the two events was the clearest. Pulsation amplitudes are given in both cases in form of binary logarithms and the scales at the different stations are not comparable as mentioned previously. In the case of the first interval, there is hardly any connection between the two events at any of the stations, in spite of some pulsation activity being present everywhere. On the other day there was a close connection between the two events, the whistler occurrence rate was, however, very low, i.e. the connection may be statistically insignificant.

Figure 6 represents the average of all the six days, synchronised to the most frequently occurring bin of the pulsation amplitudes. In this case, there is some connection between the two phenomena, the occurrence frequency of whistlers per minute increased at NCK and at NGK with increasing pulsation activity. It should be
970404
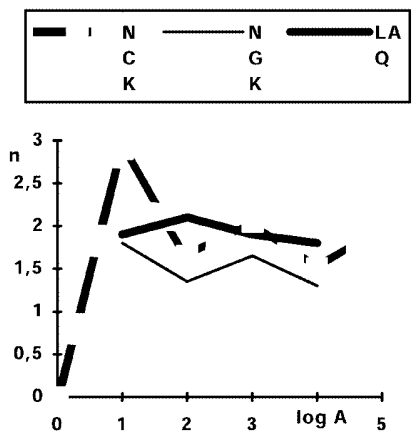

970317
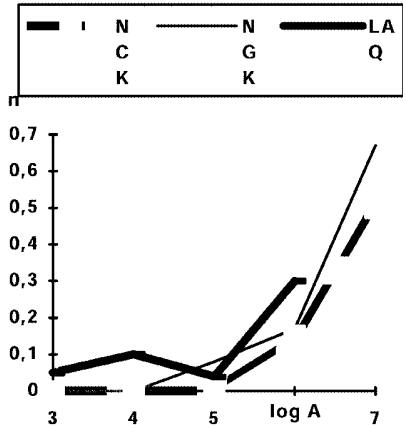

Fig. 5. Number of whistlers at the three stations versus simultaneous pulsation activity. April 4, 1997 (left) was the day with highest whistler occurrence, March 17, 1997, (right) was the day when the connection between whistlers and pulsations seemed to be best. Steps between pulsation amplitudes $(A)$ correspond to a factor of 2 (logarithmic scale), whistler numbers $(n)$ are given per minute

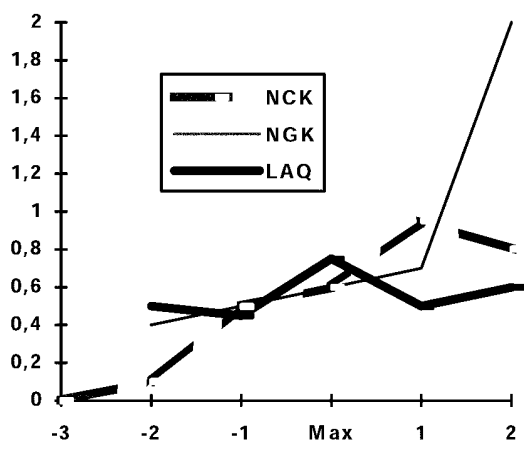

Fig. 6. Number of whistlers per minute versus pulsation activity (binary logarithm of the amplitudes) as average of all six intervals. Max means the most frequently occurring amplitude bin 
remembered that pulsation amplitudes in the bins $-2,-3$ and +2 were relatively rare, thus these values are less reliable. In spite of this, the increase of the whistler numbers is evident between pulsation amplitudes -1 and +1 , also, the increase is 50 to $100 \%$. At LAQ, there is no similar increase, the number of whistlers per minute is constant at all pulsation amplitudes. It should be remarked that the LAQ is the station at the lowest $L$ value, well outside the zone of whistler propagation, and the best connection was found at NGK, at the station with the highest $L$ value, inside the zone of whistler propagation.

In any case, there was only a slight connection if any between the occurrence frequency of whistlers and pulsation activity in the period range of field line resonances. The connection could not be significantly improved by any other choice of the method of comparison (e.g. pulsation activity versus whistler occurrence, other methods to estimate pulsation amplitudes, shorter or longer time intervals etc.).

As a shift between the occurrence of whistlers and pulsation activity cannot be excluded, we also determined a short portion (between minutes -1 and +1 ) of the cross correlation function. These functions, however, brought a somewhat surprising result. In spite of very low correlations between the two series at a lag of 0 , this value proved to be (at least a local) maximum in the majority of cases. Figure 7 shows this function for April 4, 1997, Fig. 8 the average for all the six intervals, without any weighting. On April 4, the maximum at lag 0 is higher by 0.1 to 0.3 than the adjacent values. This difference is not large, however, it is present at all three stations. The situation is not very different in the other cases, thus the average (Fig. 8) has the same pattern, with differences of 0.05 to 0.2 between lag 0 and the adjacent values. In 19 cases out of 24, the central value (lag 0) was higher than those at $\min +1$ and $\min -1$. The probability of the occurrence of such a distribution of two equally probable events is from the binomial distribution less than $0.5 \%$ in a random sample. It is an additional fact that the highest peak at lag 0 was found on April 4, 1997, when most whistlers were observed.

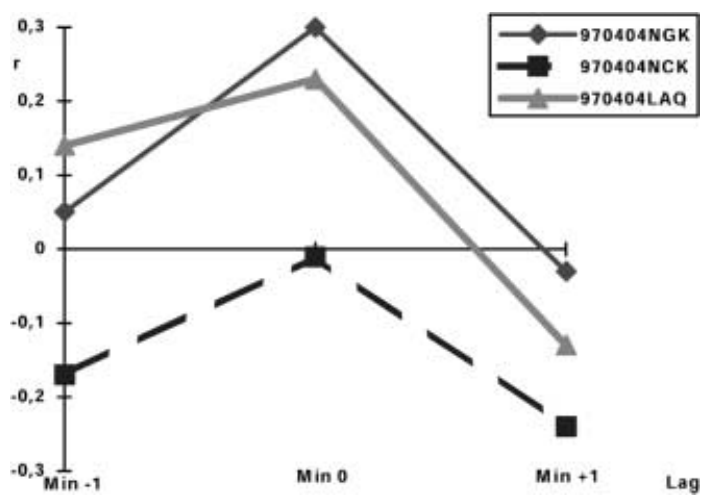

Fig. 7. Cross correlation functions between occurrence frequency of whistler and pulsation amplitudes on April 4, 1997 for lags -1 to +1

\section{Change of the pulsation spectrum at different levels of whistler activity}

In order to study all possible connections between the spectra of pulsations and the $L$ value of the propagation duct of the simultaneous whistlers, we chose the following method. Having the list of whistlers (essentially time and $L$ value), a simultaneous pulsation spectrum was selected in the nearest moment (differing by less than $3 \mathrm{~s}$ from the moment of the whistler observation). Then these spectra were distributed into four groups, with $L$ values of the whistler paths $<2.5$, $2.5-2.75,2.75-3$ and $>3$. The number of these cases was nearly the same as in Table 1, only a few pulsation data were lacking at certain stations, but the number of such cases was less than $5 \%$ of the whole. The average spectra were then computed from these groups for all the three stations. As the number of cases proved to be too small for four groups, we united the first two and the second two groups, thus finally two groups were studied. It should be remarked that the spectra for both pairs in the two groups were similar, but with higher scatter than the finally accepted average. The number of the individual spectra was 119 in the group with $L<2.75$ and 70 in the group $L>2.75$, with 1-4 missing spectra.

The average spectra for the two groups are represented in Figs. 9-11 at the three stations together with their difference. The zero line for the differences are also plotted (thin line; thick, if the pulsation amplitudes are greater at the moment of whistlers propagating at $L$ values higher than 2.75).

The average difference of the logarithms of the pulsation amplitudes (i.e. the logarithm of the ratio of the amplitudes) at moments when whistlers propagated at $L>2.75$ and $L<2.75$ are plotted in average of all the three stations (Fig. 12). (In this averaging all events were included without weighting, even those when activity in the period range of pulsations was likely to be due to noise). At periods shorter than $38 \mathrm{~s}$, the activity is higher if whistlers propagated at lower $L$ shells, above this limit, the pulsation amplitudes were greater if whistlers propagated at higher $L$ values. This value of the FLR period, $38 \mathrm{~s}$ corresponds roughly to

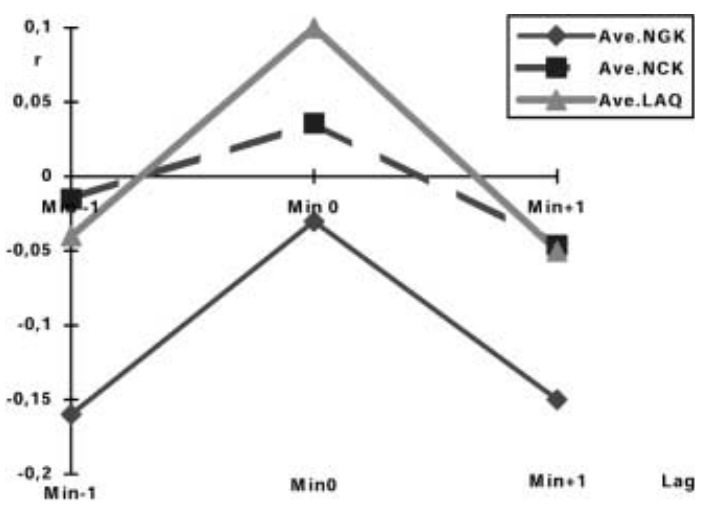

Fig. 8. Cross correlation functions between occurrence frequency of whistler and pulsation amplitudes as average of all six intervals for lags -1 to +1 


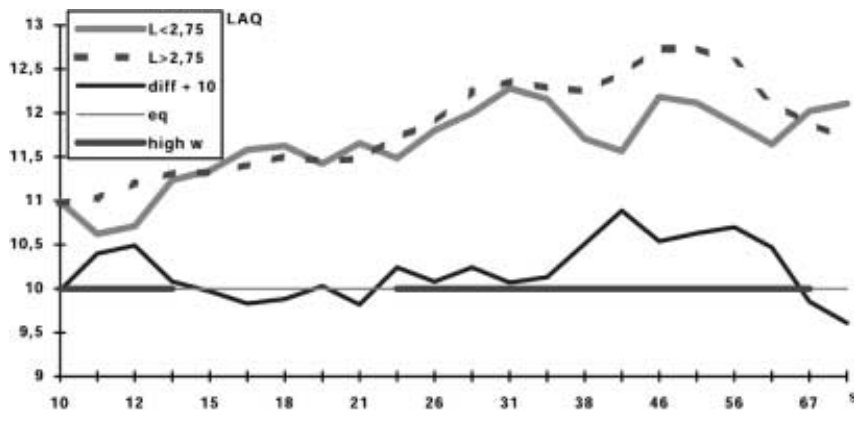

Fig. 9. Average amplitude spectra of the pulsations at the station $L A Q$ for the moments of whistler propagation at high and at low $L$ values. Amplitudes are given as ${ }^{2} \log$ A (vertical axis). The difference of the two (positive, if the amplitudes during high latitude whistlers are greater) are also plotted, the zero line is indicated, too. Thick sections (high w) refer to period ranges where pulsation amplitudes are greater if whistlers propagate at higher $L$ values

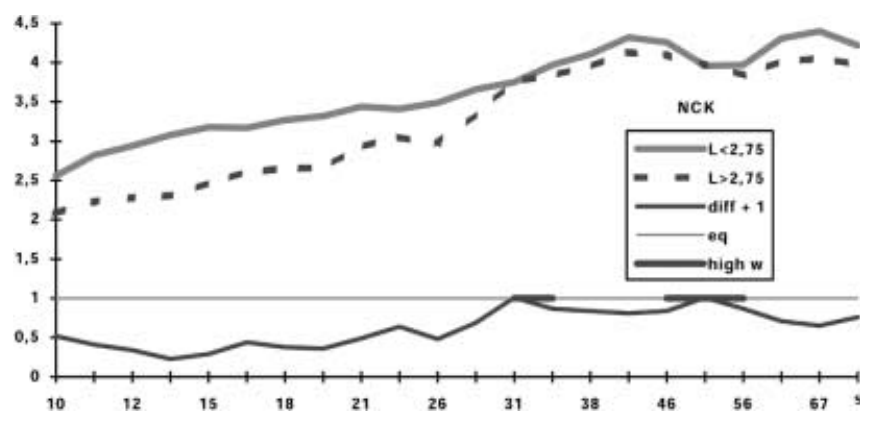

Fig. 10. The same as Fig. 9, but for the station $N C K$

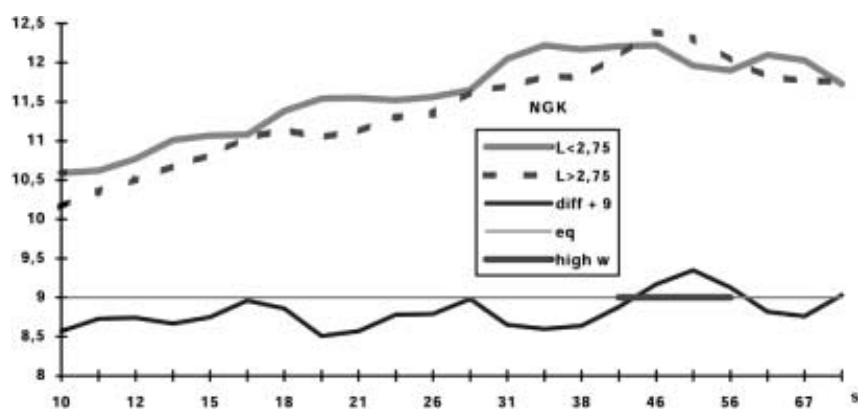

Fig. 11. The same as Fig. 9, but for the station $N G K$

the field line resonant period of the limit of the two groups of whistler propagation, i.e. to $L=2.75$. The extrema of the difference curve are found around $20 \mathrm{~s}$ (for low latitude whistlers) and around $50 \mathrm{~s}$ (high latitude whistlers), corresponding again roughly to the FLR period at limits of $L$ values where the observed whistlers propagated. Taking into account that there are several factors which make the estimation of the actual field line resonance periods inaccurate (no measurements are available for these periods in 1997, and the certainly very early time of day, when pulsation periods may be significantly shorter than later during the day, personal communication, Zieger, 1998), the correspon-

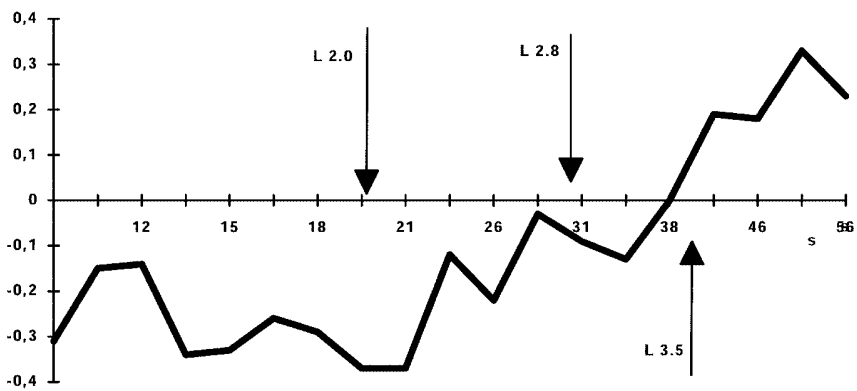

Fig. 12. Average difference at the three stations LAQ, NCK and NGK in ${ }^{2} \log A$ between pulsation amplitudes at moments when whistlers propagated at $L$ values above and below 2.75 , respectively. The periods experienced in earlier array studies at some $L$ values are indicated by arrows

dence between the two phenomena is very close. Values on the vertical axis are plotted as binary logarithms, thus a difference of 1 unit corresponds to a ratio of 2 in the amplitudes. Both extremal values mean an amplification by a factor of about 1.25 , thus the two extrema differ by a factor of 1.6 , i.e. by $60 \%$ which is considered to lie outside of the error limit.

There are two kinds of positive anomalies in Figs. 911, plotted also in Fig. 13 where the thick sections are collected from the latter figures for the three stations. The first corresponds to the maximum in the average differential spectrum, Fig. 9 with a maximum in the range around $50 \mathrm{~s}$ and upwards. The second kind is a somewhat surprising one, namely that the spectral peaks follow roughly the periods of the field line resonances (indicated very approximately by a thin line in Fig. 13). This figure is by no means a scale-correct plot, it is only an indication of this maximum near the local field line resonance. (Due to the uncertainty of the determination of these maxima, a more exact coincidence cannot be reached with the actual field line resonance periods). It is rather difficult to explain this second set of maxima. Perhaps the incoming upstream wave-type activity excites field line resonances at higher latitudes, but they also penetrate to lower $L$ values and cause local field line resonances even around $L \sim 2$. The ducts of whistler propagation may develop at lower latitudes, too, but due to a lack of sufficient incoming magnetohydro-

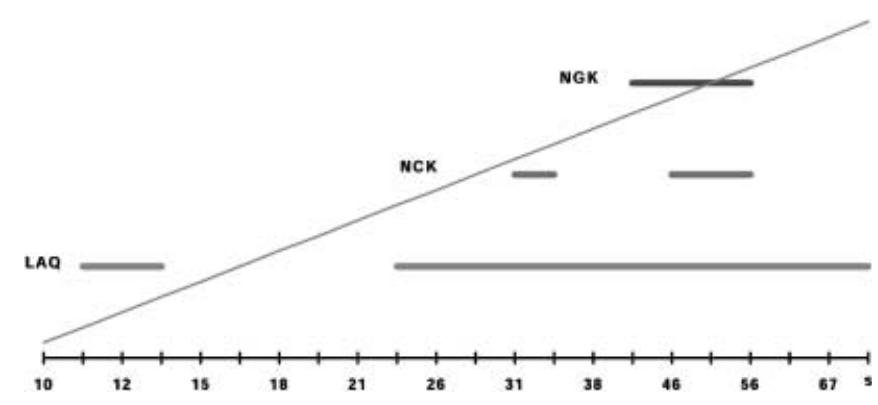

Fig. 13. Period ranges (thick lines) at the three stations, where pulsation amplitudes are greater in moments of whistlers propagating at high $L$ values than in moments of whistlers propagating at low $L$ values. The thin line connects secondary maxima to indicate that they increase with latitude similarly to field line resonance periods 
dynamic energy, at least during this very early part of the day, it cannot excite FLR.

\section{Conclusions and discussion}

The present study was carried out to investigate the connection between whistlers and (Pc3 type) pulsations at three low-latitude stations. Previously a statistical connection was found both in the position of the peak in the spectra of pulsations (the peak shifted towards longer periods if whistlers propagated at higher $L$ values) and in the activity of the two phenomena (increased pulsation activity on days with high whistler activity). In the present case, simultaneous whistler (Tihany) and pulsation measurements at three stations (L'Aquila, Nagycenk and Niemegk) were carried out during 60 intervals at $0300-0400$ UT (4-5 h LT). From a total of 60 recorded intervals, six could be more or less used in the present study when both whistlers and pulsations occurred. Concerning the two points of the previous study, the following results were obtained in this strictly simultaneous comparison:

1. The correlation between simultaneous whistler activity and pulsation activity can be confirmed, even if the connection is less close in the present case than in the previous study. It seems that the connection is rather a certain tendency for the simultaneous occurrence in longer time intervals (e.g. days) than a strict simultaneity of the two phenomena. In spite of this, a weak correlation between the two activities can be confirmed from the present study, too.

2. There is a clear shift of the amplitude spectrum toward longer periods if whistlers propagate at high $L$ values. These longer period pulsations are enhanced at all three stations. Moreover, there is a second maximum in the spectra during high- $L$ whistlers which follows quite exactly the local FLR period at the three sites. The interpretation of this second peak is not easy. Possibly the magnetohydrodynamic energy penetrating the magnetosphere at high $L$ values may also contain some signal in the short period range which is locally amplified at the resonance frequency. On the other hand, low- $L$ whistlers are not accompanied by pulsations at higher latitudes. That would mean that not only magnetohydrodynamic waves, coming from the interplanetary medium, but also rapid magnetic variations at high latitude, propagating along the ionosphere can excite field line resonance at low to very low latitude. For DP2-type variations Kikuchi et al. (1996) found a very high degree of coherence for magnetic features generated at high latitude all the way from subauroral latitudes down to the dip-equator. They proposed an ionospheric signal propagation.

Evidently the present material is insufficient to fully support this possibility, nevertheless, we do not see another explanation for the observed FLR signal simultaneously with whistlers propagating at high $L$-values. Some further support for this supposition is due to the fact that at very low latitudes, near to the equator pulsations are somewhat better correlated to pulsations at distant high latitude sites (e.g. both Dhargapur in India and Bac Lieu in Vietnam, just at the geomagnetic equator are better correlated with Niemegk than with Nagycenk, Verö et al., 1991, 1992).

Up to now, two mechanisms were proposed to explain the pulsation - whistler correlation. One of them (Smith et al., 1998) is based on modulation of the whistler mode wave growth rates by field line resonances, resulting in type II QP events, while they supposed type I QP events to be due to upstream waves coming from the interplanetary space. Verö et al.'s (1997) idea was that the occurrence frequency of whistler ducts and shells of field line resonances similarly changes according to the actual magnetospheric conditions. When comparing the two possibilities, some points should be taken into account.

1. In Smith et al.'s (1998) sample, only type I QPs are accompanied with geomagnetic pulsations and they are not connected with whistlers.

2. Smith et al.'s (1998) events were observed in the polar region, while ours are from low-latitude stations.

3 . We found only a slight correlation for simultaneous whistlers and pulsations, while the correlation with long time averages of these activities was essentially higher.

Waves responsible for the Type II QPs observed by Smith et al. (1998) at high latitudes may propagate to lower $L$-values and if the conditions are favourable for field line resonances there, then they may get excited as the present study showed it (Fig. 13), and the same conditions may also be favourable for the propagation of whistlers at low latitudes. Thus, we do not think that the two mechanisms are conflicting, they are rather supplementary ones, one is characteristic at higher, the other (ours) at lower latitudes. In any case, the correlation between simultaneous events found in this study may result from the mechanism described by Smith et al. (1998). The long lifetime of the tendency of the common occurrence of both whistler ducts and field line shells can more easily be explained by a common source effect of the two phenomena than by direct influence, as in Smith et al.'s (1998) mechanism. The noise level in the short period range of our stations did not permit us to study short period signals observed by Smith et al. (1998) in detail, however, no corresponding signals were detected in our sample by visual inspection in spite of measurements in the early dawn hours when they observed such activity.

In summary, the low-latitude magnetosphere seems to have sometimes more inclination both for whistler propagation (development of whistler ducts) and for FLR type pulsations (shells of field lines). Such structures may be present on time scales of about one hour to one day, and due to the common influencing factor, the two events are correlated on hourly to daily time scales. For shorter intervals, the instantaneous response is less evident but it still exists, perhaps by a mechanism similarly to that supposed by Smith et al. (1998). Moreover, the whole of the magnetosphere is not capable to produce such events, but only some part of 
it, the part being again the same for the two events, i.e. if whistlers propagate at higher latitudes then pulsations have longer periods, too. Moreover, we repeatedly found some indication of a connection between highlatitude and (relatively) low-latitude events which, however, need further verification, similarly to all connections described in the present study. This can only be made by synchronous long-term recording of the two phenomena at several sites.

Acknowledgements. The authors thank two referees for valuable comments and suggestions. The present investigation was supported in Hungary by a state grant OTKA T 19568, as well as by Italian-Hungarian (TéT) and Czech-Hungarian (Academies of Sciences) scientific cooperation grants, a part of that also by grant 205/99/0915 of the Grant Agency of the Czech Republic.

Topical Editor G. Chanteur thanks M. J. Engebretson and another referee for their help in evaluating this paper.

\section{References}

Cz. Miletits, J., J. Verö, J. Szendröi, P. Ivanova, A. Best, and M. Kivinen, Pulsation periods at mid-latitudes: a seven-station study, Planet. Space Sci., 38, 85-95, 1990.

Helliwell, R. A., Whistlers and related ionospheric phenomena, Stanford University Press, Stanford, California, 1965.

Kikuchi, T. H., H. Lühr, O. Kitamura, O. Saka, and K. Sclegel, Direct penetration of the polar electric field to the equator during a DP2 event as detected by the auroral and equatorial chains and the EISCAT radar, J. Geophys. Res., 101, 17 161$17173,1996$.

Lee, M. A., Coupled hydromagnetic wave excitation and ion acceleration upstream of the earth's bow shock, J. Geophys. Res., 87, 5063-5080, 1982.

Maeda, K., and I. Kimura, A theoretical investigation of the propagation path of the whistling atmospherics, Rep. Ionosph. Space. Res. Japan, 10, 105-123, 1956.

Orr, D., Magnetic pulsations in the magnetosphere: a review, J. Atmos. Terr. Phys., 35, 1-50, 1978.

Poulter, E. M., M. K. Allan, G. J. Bailey, and R. J. Moffett, Radial plasma drifts deduced from VLF whistler mode signals: a modeling study, Planet. Space. Sci., 32, 525-533, 1984.

Smith, A. J., M. J. Engebretson, E. M. Klatt, U. S. Inan, R. L. Arnoldy, and H. Fukunishi, Periodic and quasi-periodic ELF/
VLF emissions observed by an array of Antarctic stations, J. Geophys. Res., 103, 23 611-23 620, 1998.

Smith, R. L., Guiding of whistlers in a homogenous medium, J. Res. NBS-D, Radio Propagation, 64D, 505-508, 1960.

Smith, R. L., and J. J. Angerami, Magnetospheric properties deduced from Ogo 3 observations of ducted and non-ducted whistlers, J. Geophys. Res., 73, 1-20, 1968.

Smith, R. L., R. A. Helliwell, and I. Yabroff, A theory of trapping of whistlers in field-aligned columns of enhanced ionization, J. Geophys. Res., 65, 815-823, 1960.

Strangeways, H. J., The effect of multiduct structure on whistlermode wave propagation, J. Atmos. Terr. Phys., 44, 901-912, 1982.

Strangeways, H. J., An investigation of the propagation of whistlers in magnetospheric ducts by means of ray-tracing, curve-fitting and direction-finding techniques, $\mathrm{PhD}$ Thesis, University of Southampton, UK, 1976.

Takahashi, O., K. Ohta, and M. Hayakawa, On the structure of ducts for mid-latitude whistlers and their ionospheric transmission as deduced from the ground-based direction finding, PAGEOPH, 140, 519-535, 1993.

Tarcsai, Gy., Routine whistler analysis by means of accurate curve fitting, J. Atmos. Terr. Phys., 37, 1447-1458, 1975.

Verö, J., Solar cycle effect on Pc3 geomagnetic pulsations, J. Geophys. Res., 101, 2461-2465, 1996.

Verö, J., and F. W. Menk, Damping of geomagnetic pulsations at high F2-layer electron concentrations, J. Atmos. Terr. Phys., 48, 231-245, 1986.

Verö, J., L. Holló, and B. P. Singh, Geomagnetic pulsations at lowand mid-latitudes, Acta Geod., Geophys. Mont. Hung., 26, 253263, 1991.

Verö, J., Le Minh Triet, and J. Szendröi, Pulsations at the geomagnetic equator, Acta Geod., Geophys. Mont. Hung., 27, 177-184, 1992.

Verö, J., L. Holló, P. Bencze, and F. Märcz, Whistler ducts and geomagnetic pulsation resonant field line shells near $L=2$ - are they identical? J. Atmos. Terr. Phys., 59, 1855-1864, 1997.

Verö, J., H. Lühr, M. Vellante, I. Best, J. Strestik, J. Cz. Miletits, L. Holló, J. Szendröi, and B. Zieger, Upstream waves and field line resonance: simultaneous presence and alternation in Pc3 pulsation events, Ann. Geophysicae, 16, 34-48, 1998.

Walker, A. D. M., The theory of whistler propagation, Rev. Geophys. Space Phys., 14, 629, 1976.

Walker, A. D. M., Formation of whistler ducts, Planet. Space Sci., 26, 375-379, 1978.

Yumoto, K., Generation and propagation mechanisms of lowlatitude magnetic pulsations, J. Geophys., 60, 79-105, 1986. 